\title{
On bipartite graphs and the Fibonacci numbers
}

\author{
Fatih Yılmaz $^{1}$ and Pinar Eldutar ${ }^{2}$ \\ ${ }^{1}$ Department of Mathematics, Polatlı Art and Science Faculty, \\ Ankara Haci Bayram Veli University, 06900 Ankara, Turkey \\ e-mail: fatih.yilmaz.edu@gmail.com \\ 2 Department of Mathematics, \\ Gazi University, 06900 Ankara, Turkey \\ e-mail: peldutaregmail.com
}

Received: 25 March 2019

Revised: 14 November 2019

Accepted: 18 November 2019

\begin{abstract}
In this short note, we consider adjacency matrices of ladder graphs. Then we obtain permanental polynomials, eigenvalues and some other properties of adjacency matrix of the graph.
\end{abstract}

Keywords: Permanental polynomial, Fibonacci numbers, Eigenvalue.

2010 Mathematics Subject Classification: 15A15, $11 \mathrm{~B} 39$.

\section{Preliminaries}

Let $G=(V, E)$ be an undirected graph with vertex set $V=\left(v_{1}, v_{2}, \ldots, v_{n}\right)$ and the edge set $E$. The adjacency matrix $A(G)=A=\left[a_{i j}\right]$ is an $n$-square matrix of zeros and ones for which $a_{i j}=1$ iff $v_{i}$ is adjacent to $v_{j}$ (which means there is an edge between $v_{i}$ and $v_{j}$ ).

It is clear that the determinant of an $n \times n$ matrix $\mathcal{A}=\left(a_{i j}\right)$ may be given by

$$
\operatorname{det}(\mathcal{A})=\sum_{\sigma \in \mathcal{S}_{n}} \operatorname{sgn}(\sigma) \prod_{i=1}^{n} a_{i \sigma(i)},
$$

where $\mathcal{S}_{n}$ represents the symmetric group of degree $n$. Analogously, if one omits the sign pattern which the determinant involves, we get the permanent of $\mathcal{A}$ defined by

$$
\operatorname{per}(\mathcal{A})=\sum_{\sigma \in \mathcal{S}_{n}} \prod_{i=1}^{n} a_{i \sigma(i)} .
$$


In general, permanents of matrices have noteworthy combinatorial significance. Especially, the permanents of $\{0,1\}$ matrices enumerate matchings in bipartite graphs.

The characteristic polynomial of adjacency matrix of a graph is defined with

$$
\Omega(G, \lambda)=\operatorname{det}(\lambda I-A(G))
$$

The characteristic polynomial of graphs and its applications are intensively studied (see $[3,4,11]$ and references therein).

The permanental polynomial of $A$ is

$$
\Pi(G, \lambda)=\operatorname{per}(\lambda I-A(G))
$$

where per denotes the permanent of the matrix. It is considered that the permanental polynomial was first studied by Turner [15]. The author takes into account a graph polynomial which generalizes both the permanental polynomial and the characteristic polynomial. In chemistry, the permanental polynomial was first considered at 1981 by Kasum et al [8]. The authors showed the relationships between the permanental polynomial and the structure of conjugated molecules. In Chemical Graph Theory, the skeleton of a hydrocarbon molecule can be represented by a simple graph and the adjacency matrix of a simple graph can be represented by a symmetric $\{0,1\}$ matrix.

The Kronecker product, also called as tensor product, is a way of matrix multiplication. Let us consider two matrices $A$ and $B$ of sizes $m \times n$ and $s \times t$, respectively. Their Kronecker product is defined by

$$
A \otimes B=\left(\begin{array}{cccc}
a_{11} B & a_{12} B & \cdots & a_{1 n} B \\
a_{21} B & a_{22} B & \cdots & a_{2 n} B \\
\vdots & \vdots & \ddots & \vdots \\
a_{m 1} B & a_{m 2} B & \cdots & a_{m n} B
\end{array}\right)
$$

Here we want to remember some useful properties of Kronecker product of matrices [17], i.e.;

(i) For $A=\left[a_{i j}\right]_{m \times m}$ and $B=\left[b_{i j}\right]_{n \times n}$;

$$
|A \otimes B|=|A|^{n} \cdot|B|^{m}=|B \otimes A|
$$

(ii) For any square $A$ and $B$ matrices, if $A^{-1}$ and $B^{-1}$ exist, then

$$
(A \otimes B)^{-1}=A^{-1} \otimes B^{-1} .
$$

There exists a vast literature that studies the various properties of graphs and their relationships. Merris et al. [9] are the first to systematically study the permanental polynomials and they proved that the coefficient of the permanental polynomial satisfies that

$$
(-1)^{i} b_{i}=\sum_{H} 2^{k(H)}
$$

where the sum ranges over all subgraphs $H$ on $i$ vertices which components are single edges or cycles, and $k(H)$ is the number of cycles. Based on this result, similarly to the technique of 
computing the characteristic polynomial of a graph in terms of subgraphs [12], Borowiecki and Jozwiak [1] studied the relationship between the permanental polynomial of a dimultigraph and certain subgraphs.

The well-known Fibonacci sequence is defined by the recurrence relation $F_{n+1}=F_{n}+F_{n-1}$ with initial conditions $F_{0}=0$ and $F_{1}=1$. In [7], Kalman considered the sequence

$$
a_{n+k}=c_{0} a_{n}+c_{1} a_{n+1}+\cdots+c_{k-1} a_{n+k-1},
$$

where $c_{0}, c_{1}, \ldots, c_{k-1}$ are constants and the author generalized the sequence by companion matrix, as below:

$$
\mathcal{H}^{n}\left(\begin{array}{c}
a_{0} \\
a_{1} \\
\vdots \\
a_{k-1}
\end{array}\right)=\left(\begin{array}{c}
a_{n} \\
a_{n+1} \\
\vdots \\
a_{n+k-1}
\end{array}\right)
$$

where

$$
\mathcal{H}=\left(\begin{array}{ccccc}
c_{0} & c_{1} & \cdots & c_{k-2} & c_{k-1} \\
1 & 0 & \cdots & 0 & 0 \\
0 & 1 & \cdots & 0 & 0 \\
\vdots & \vdots & \ddots & \vdots & \vdots \\
0 & 0 & 0 & 1 & 0
\end{array}\right)
$$

This paper is concerned with ladder graphs which can be obtained as the Cartesian product of two path graphs. Moreover, this paper is partly presented at the conference given by [5].

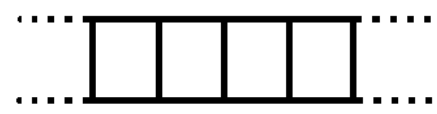

The adjacency matrix of the graph, given by (1.3), is an $n$-square $(n=2 k)$ block matrix

$$
A=A(G)=\left(\begin{array}{c|c}
B_{k} & I_{k} \\
\hline I_{k} & B_{k}
\end{array}\right),
$$

where

$$
B_{k}=\left[b_{i j}\right]= \begin{cases}1, & \text { for } i=j+1 \text { and } j=i+1 \\ 0, & \text { otherwise }\end{cases}
$$

and $I_{k}$ is the identity matrix of order $k$.

Example 1.1. For instance, if $k=3(n=6)$, the adjacency matrix is:

$$
A(G)=\left(\begin{array}{cccccc}
0 & 1 & 0 & 1 & 0 & 0 \\
1 & 0 & 1 & 0 & 1 & 0 \\
0 & 1 & 0 & 0 & 0 & 1 \\
1 & 0 & 0 & 0 & 1 & 0 \\
0 & 1 & 0 & 1 & 0 & 1 \\
0 & 0 & 1 & 0 & 1 & 0
\end{array}\right)
$$


It is known that a matching (for graphs) is a set of edges which satisfies the property that no vertex is incident with more than one edge in the set. Moreover, a matching is perfect iff every vertex is incident with exactly one edge of the matching [16]. In other words, if a graph, with even $n=|V|$ numbers, has a matching with $n / 2$ edges, it is called a perfect matching graph [6] and the number of possible perfect matchings of a graph is the perfect matching number, or Kekule number. In this note, we consider ladder graphs whose adjacency matrix is $\{0,1\}$ matrix. By using the fact, the permanents of $\{0,1\}$ matrices enumerate matchings in bipartite graphs, we show that the perfect matchings of the graph corresponds to the square of the Fibonacci numbers. Moreover, we get additional spectecular properties of the graph.

\section{Main results}

Theorem 2.1. For $k>2$, the permanental polynomial of $A(G)$ is

$$
P_{2 k+2}(x)=\left(x^{2}+3\right) P_{2 k}(x)-P_{2 k-2}(x)+2(-1)^{k-1}
$$

with initial conditions $P_{6}(x)=x^{6}+7 x^{4}+15 x^{2}+9$ and $P_{4}(x)=x^{4}+4 x^{2}+4$.

Proof. Using the Principle of Mathematical Induction (PMI), it is clear that the theorem holds for $k=3$. Suppose that it verifies for $k=t$. Then, we need to verify for $k=t+1$. By considering the same way with Kalman [7], we can write (for $k>2$ ),

$$
\left(\begin{array}{cc}
x^{2}+3 & -1 \\
1 & 0
\end{array}\right)\left(\begin{array}{c}
P_{n} \\
P_{n-2}
\end{array}\right)=\left(\begin{array}{c}
P_{n+2}+2(-1)^{k} \\
P_{n}
\end{array}\right) .
$$

By using (2.2), it is easy to see that it holds for $k=t+1$.

From the theorem, we have the following result.

Corollary 2.2. The permanent of the adjacency matrix $A(G)$, given in (1.4), is

$$
\operatorname{per} A(G)=F_{k}^{2}
$$

where $F_{k}$ is the $k$-th Fibonacci number.

Proof. For $x=0$ at (2.1), the proof is clear.

Since the adjacency matrix of the graph is a block matrix, let us remind the following property for block matrices, without proof.

Let us define $n$-square $(n=2 k)$ matrix $E_{n}$ as below:

$$
E_{n}=\left(\begin{array}{l|l}
I_{k} & I_{k} \\
\hline I_{k} & -I_{k}
\end{array}\right),
$$

where $I_{k}$ is a $k$-square identity matrix. Then we have the following lemmas.

Lemma 2.3 ([5]). $\operatorname{det} E_{n}=(-2)^{k}$. 
Proof. It is obvious that $E_{n}$ can be rewritten as below:

$$
E_{n}=\left(\begin{array}{cc}
1 & 1 \\
1 & -1
\end{array}\right) \otimes I_{k}
$$

by (1.1), the proof can be seen easily.

Lemma 2.4 ([5]). The inverse of $E_{n}$ is

$$
E_{n}^{-1}=\frac{1}{2}\left(\begin{array}{l|l}
I_{k} & I_{k} \\
\hline I_{k} & -I_{k}
\end{array}\right) .
$$

Proof. It can be verified using (1.2).

Firstly, let us consider $n$-square block-matrix $\left(U=\left[u_{i j}\right]\right.$ and $\left.V_{k}=\left[v_{i j}\right]\right)$,

$$
M_{n}=\left(\begin{array}{l|l}
U_{k} & 0 \\
\hline 0 & V_{k}
\end{array}\right),
$$

where

$$
\left[u_{i j}\right]= \begin{cases}1, & \text { for } i=j, i=j+1 \text { and } j=i+1 \\ 0, & \text { otherwise }\end{cases}
$$

and

$$
\left[v_{i j}\right]=\left\{\begin{array}{cl}
1, & \text { for } i=j+1 \text { and } j=i+1 \\
-1 & \text { for } i=j \\
0, & \text { otherwise }
\end{array} .\right.
$$

Theorem 2.5. The matrices $A(G)$ and $M_{n}$ are similar.

Proof. By matrix multiplication, it can be seen that $M_{n}=E_{n}^{-1} A(G) E_{n}$ provides. So the proof is completed.

Lemma 2.6 ([10]). The eigenvalues of the matrix

$$
\left(\begin{array}{cccc}
0 & 1 & & \\
1 & 0 & \ddots & \\
& \ddots & \ddots & 1 \\
& & 1 & 0
\end{array}\right)
$$

are

$$
\lambda_{k}=-2 \cos \frac{k \pi}{n+1}, \quad k=1,2,3, \ldots, n .
$$

Theorem 2.7. The eigenvalues of $M_{n}$ are

$$
\begin{aligned}
& \lambda_{j}\left(U_{k}\right)=\left(1-2 \cos \frac{\pi j}{k+1}\right), \\
& \lambda_{j}\left(V_{k}\right)=\left(-1-2 \cos \frac{\pi j}{k+1}\right),
\end{aligned}
$$

where $j=1,2, \ldots, k$. 
Proof. Since the matrices $U_{k}$ and $V_{k}$ are tridiagonal matrices, by exploiting the well-known Chebyshev polynomial properties, it is easy to compute the eigenvalues by using [2] and [10].

Hereby, similar matrices have the same eigenvalues, trace, determinant, rank, Jordan form and number of independent eigenvectors [14].

\section{Theorem 2.8.}

$$
\operatorname{det} A(G)=\prod_{j=1}^{k}\left(1-2 \cos \frac{\pi j}{k+1}\right)\left(-1-2 \cos \frac{\pi j}{k+1}\right)
$$

where $j=1,2, \ldots, k$.

Proof. Since the characteristic polynomials of $A(G)$ and $M_{n}$ are equal, then their determinants are also the same. The eigenvalues of $U_{k}$ and $V_{k}$ are, respectively:

$$
\begin{aligned}
& \lambda_{j}\left(U_{k}\right)=\left(1-2 \cos \frac{\pi j}{k+1}\right), \\
& \lambda_{j}\left(V_{k}\right)=\left(-1-2 \cos \frac{\pi j}{k+1}\right) .
\end{aligned}
$$

So the proof is completed.

\section{Acknowledgements}

The authors present their appreciations to referees for providing their valuable suggestions.

\section{References}

[1] Borowiecki, M., \& Jozwiak, T. (1982). Computing the permanental polynomial of a multigraph, Discuss. Math., 5, 9-16.

[2] Cahil, N. D., D'Errico, J. R., \& Spence, J. P. (2003). Complex factorizations of the Fibonacci and Lucas numbers, The Fibonacci Quarterly, 41 (1), 13-19.

[3] Codenotti, B., \& Resta, G. (2002). Computation of spare circulant permanents via determinants, Linear Alg. Appl., 355, 15-34.

[4] Cvetkocic, D. M., Doob, M., Gutman, I., \& Torgasev, A. (1988). Recent Results in the Theory of Graph Spectra, North-Holland, Amsterdam, p. 123.

[5] Eldutar, P., \& Yilmaz, F. (2016). On linear algebra of adjacency matrices for one type of graph, ICAMA-2016, Ankara, Turkey. 
[6] Hosoya, H. (2004). Sequences of polyomino and polyhex graphs whose perfect matching numbers are Fibonacci or Lucas numbers: The Golden family graphs of a new category, Forma, 19, 405-412.

[7] Kalman, D. (1982). Generalized Fibonacci numbers by matrix methods, The Fibonacci Quarterly, 20 (1), 73-76.

[8] Kasum, D., Trinajstic, N., \& Gutman, I. (1981). Chemical graph theory. III, On the permanental polynomial, Croat. Chem. Acta, 54, 321-328.

[9] Merris, R., Rebman K. R., \& Watkins, W. (1981). Permanental polynomials of graphs, Lin. Algebra Appl, 38, 273-288.

[10] Rimas, J. (2006). On computing of arbitrary positive integer powers for one type of symmetric tridiagonal matrices of even order-II, Applied Mathematics and Computation, $172,245-251$.

[11] Rosenfeld, V. R., \& Gutman, I. (1989). A novel approach to graph polynomials, MatchCommun. Math. Comput. Chem., 24, 191-199.

[12] Schwenk, A. J. (1974). Computing the Characteristic Polynomial of a Graph, SpringerVerlag, Berlin, pp. 153-172.

[13] Shannon, A., Turner, J., \& Atanassov, K. (1991). A generalized tableau associated with colored convolution trees, Discrete Mathematics, 92 (1-3), 329-340.

[14] Strang, G. (2009). Introduction to Linear Algebra, Wellesley-Cambridge Press.

[15] Turner, J. (1968). Generalized matrix functions and the graph isomorphism problem, SIAM J. Appl. Math., 16, 520-526.

[16] Vasudev, C. (2006). Graph Theory with Applications, New Age International Limited Publishers, New Delhi.

[17] Zhang, H., \& Ding, F. (2013). On the Kronecker products and their applications, Hindawi Publishing Corporation Journal of Applied Mathematics, Article ID 296185. 